\title{
EXISTENCE AND CONJUGACY OF \\ CARTAN SUBALGEBRAS OF JORDAN ALGEBRAS
}

\section{OTTMAR LOOS}

ABSTRACT. It is shown that a finite-dimen sional Jordan algebra over a field $k$ of characteristic $\neq 2$ contains Cartan subalgebras, and that any two Cartan subalgebras are conjugate by an inner automorphism provided $k$ is algebraically closed.

Let $\mathcal{Y}$ be a finite-dimensional unital (linear) Jordan algebra over a field $k$ of characteristic $\neq 2$. The purpose of this note is to complete N. Jacobson's theory of Cartan subalgebras (cf. [3]) by proving

(i) $\Im$ contains Cartan subalgebras;

(ii) if $k$ is algebraically closed then any two Cartan subalgebras are conjugate by an inner automorphism of $\mathfrak{\Im}$;

(iii) if $k$ is infinite then every Cartan subalgebra contains associator regular elements.

The proof of (ii) is essentially the same as Jacobson's, once we know that the Lie algebra of the inner automorphism group contains all inner derivations. The existence of Cartan subalgebras was proved for infinite fields in [3] and for finite fields with sufficiently many elements in [2]. Our proof of (i) uses (ii) and imitates the corresponding proof for the existence of Cartan subgroups of algebraic groups [1]. These methods seem to break down for quadratic Jordan algebras over fields of characteristic 2, mainly due to the fact that the inner automorphism group is no longer smooth (in the sense of group schemes). However, in the broader framework of Jordan pairs, there is a satisfactory theory of Cartan subpairs over fields of arbitrary characteristic (cf. [4]).

We denote by $x y=R_{x}(y)$ the multiplication in $\Im$, by $U_{x}=2 R_{x}^{2}-R_{x^{2}}$ the quadratic representation, and let

$$
U_{x, y}=U_{x+y}-U_{x}-U_{y}=2\left(R_{x} R_{y}+R_{y} R_{x}-R_{x y}\right) .
$$

Received by the editors May 14, 1974 .

AMS (MOS) subject classifications (1970). Primary 17C10.

Key words and phrases. Jordan algebra, Cartan subalgebra. 
Throughout, $K$ will be an algebraic closure of $k$ and $k_{s}$ the separable closure of $k$ in $K$. We imbed $\Im$ into $\Im_{K}=\Im_{\otimes_{k}} K$ via $x \mapsto x \otimes 1$. Our reference for algebraic groups is [1]. The Lie algebra (resp. connected component of the identity) of an algebraic group $G$ is denoted $L(G)$ or $g$ (resp. $G^{0}$ ). Recall that $L(G)=L\left(G^{0}\right)$ and $G^{0}$ is defined over $k$ provided $G$ is (see [1, pp. 86, 118]).

The inner structure group of $\Im$ is the subgroup $G$ of $G L\left(\Im_{K}\right)$ generated by all $U_{x}, x \in \Im_{K}$ invertible. By [1, p. 106], $G$ is a connected algebraic group, defined over $k$. Its Lie algebra $g=L(G)$ contains all $R_{y}, y \in \Im_{K}$, since $2 R_{y}$ is the derivative of $U_{x}$ at $x=1$ in direction $y$. For $g \in G$ let

$$
\theta(g)=U_{g(1)}^{-1} g \text {. }
$$

Then $\theta$ is an automorphism of period 2 of $G$ which is defined over $k$, and we have

$$
\begin{aligned}
& U_{g(x)}=g U_{x} \theta(g)^{-1}, \\
& \theta\left(U_{x}\right)=U_{x}^{-1},
\end{aligned}
$$

for all invertible $x \in \Im_{K}$. Let $G_{\theta}$ be the fixed point set of $\theta$ in $G$, let $L(\theta)$ be the automorphism of $g$ induced by $\theta$, and let $g=g_{+} \oplus g_{-}$be the decomposition of $g$ into the $( \pm 1)$-eigenspaces of $L(\theta)$. Clearly $g_{+}$is a subalgebra of $g$, and we have $\left[g_{+}, g\right] \subset g_{-}$and $\left[g_{-}, g_{-}\right] \subset g_{+}$. From (3) we get by differentiating

$$
L(\theta)\left(R_{x}\right)=-R_{x} \in g_{-}
$$

and hence

$$
D_{x, y}=\left[R_{x}, R_{y}\right] \in g_{+},
$$

for all $x, y \in \mathfrak{J}_{K}$. The $D_{x, y}$ are called inner derivations.

Lemma 1. $G_{\theta}$ is defined over $k$, and $L\left(G_{\theta}\right)=g_{+}$.

Proof. Let $A=G \times(\mathrm{Z} / 2 \mathrm{Z})$ be the semidirect product with the nontrivial element of $\mathrm{Z} / 2 \mathrm{Z}$ acting on $G$ via $\theta$. Then $A$ is an algebraic group defined over $k$, and $s=(e, \theta)$ is a $k$-rational point of $A$ which is moreover semisimple since it is of order two and char $(k) \neq 2$. By $[1$, p. 225], the centralizer $Z_{G}(s)$ of $s$ in $G$ is defined over $k$, and its Lie algebra is the centralizer $Z_{g}(s)$ of $s$ in $g$. From the definition it is clear that $Z_{G}(s)=G_{\theta}$ and $Z_{g}(s)=g_{+}$.

The inner automorphism group of $\mathcal{J}$ is defined by $H=\{g \in G: g(1)=1\}$. Clearly, $H$ is $k$-closed, and by (1) it is contained in $G_{\theta}$. 
Lemma 2. $H$ is of finite index in $G_{\theta}$, and hence we have $H^{0}=\left(G_{\theta}\right)^{0}$ and $L(H)=9_{+}$. In particular, $L(H)$ contains all inner derivations. Moreover, $H$ is defined over $k$.

Proof. Let $X=\left\{x \in \mathfrak{S}_{K}: U_{x}=\mathrm{Id}\right\}$. Then $X$ is an algebraic variety, $G_{\theta}$ acts on $X$ by (2), and $H$ is the isotropy group of 1 in $G_{\theta}$. We show that $X$ is finite by proving that the tangent space $T_{x}(X)$ is zero, for every $x \in X$. Indeed, $y \in T_{x}(X)$ if and only if $U_{x, y}=0$, and this implies $0=U_{x, y}(x)=$ $2 x^{2} y=2 y$ since $x^{2}=U_{x}(1)=1$. By $\left[1\right.$, p. 86] we have $\left(G_{\theta}\right)^{0} \subset H$ which proves that $H^{0}=\left(G_{\theta}\right)^{0}$ is defined over $k$ and $L(H)=L\left(G_{\theta}\right)=g_{+}$by Lemma 1. Since $H$ is obviously stable under the Galois group of $k_{s}$ over $k$ it follows that $H$ is defined over $k[1, \mathrm{p} .57]$.

Lemma 3. Suppose $k=K$ is algebraically closed. Let (S be a subalgebra of $\mathfrak{S}$ containing $\Sigma_{i=1}^{r} \mathfrak{S}_{i i}$ where $\mathfrak{S}=\Sigma \mathfrak{S}_{i j}$ is the Peirce decomposition of $\mathfrak{S}$ with respect to a supplementary set $\left\{e_{1}, \cdots, e_{r}\right\}$ of orthogonal idempotents. Then the orbit map $a: H^{0} \times \mathfrak{S} \rightarrow \mathfrak{S}$ is dominant and separable, and hence the orbit of (S. under $H^{0}$ contains an open dense subset of $\mathfrak{S}$ (in the Zariski topology).

Proof. By $[1$, p. 75] it suffices to show that the differential of $\alpha$ is surjective at some point of $H^{0} \times \mathfrak{S}$. Let $x=\Sigma \lambda_{i} e_{i}$ where the $\lambda_{i} \in k$ are such that $\lambda_{i} \neq \lambda_{j}$ for $i \neq j$. By Lemma 2 , the tangent space of $H^{0} \times(\mathbb{S}$ at $a=(\mathrm{Id}, x)$ is $g_{+} \times \mathbb{S}$, and $(d \alpha)_{a}$ is given by

$$
(d a) \underset{a}{ }(D, y)=D(x)+y,
$$

for $(D, y) \in g_{+} \times$. Hence we only have to show that all $\mathfrak{S}_{i j}$ for $i \neq j$ are contained in the image of $(d \alpha)_{a}$. But if $z \in \widetilde{S}_{i j}$ then by Lemma 2, $D_{z, x} \in g_{+}$, and an easy computation using the Peirce rules shows

$$
D_{z, x}(x)=z x^{2}-(z x) x=1 / 4\left(\lambda_{i}-\lambda_{j}\right)^{2} z .
$$

This proves the lemma.

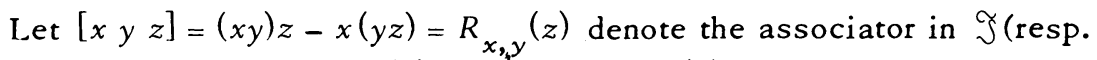
$\widetilde{\Im}_{K}$ ). For $x \in \Im_{K}$ let $T^{n}+c_{1}(x) T^{n-1}+\cdots+c_{n}(x)$ be the characteristic polynomial of $R_{x, x}$. Then the $c_{i}$ are polynomial functions on $\mathfrak{J}_{K}$ which are defined over $k$. Let $m$ be the largest integer such that $c_{n-i}=0$ for $i<m$. An element $x$ is called (associator) regular if $c_{n-m}(x) \neq 0$. (If $k$ is finite it may happen that $c_{n-m}(x)=0$ for all $x \in \mathfrak{J}_{\text {.) }}$ The set $\mathfrak{J}_{\text {reg }}$ of regular elements of $\mathfrak{\Im}_{K}$ is open and dense.

Recall that a Jordan algebra is associator nilpotent if there exists an 
integer $n$ such that any product of $n$ of the $R_{x, y}$ 's vanishes. A subalgebra (5) of $\mathfrak{S}$ is called a Cartan subalgebra if it is associator nilpotent and equal to its own normalizer in the sense that [ 다 $\sqrt{5} z] \subset$ ( 5 implies $z \in \mathbb{E}$. If $x$ is regular then

$$
B_{x}=\left\{y \in \breve{S}: R_{x, x}^{n}(y)=0 \text { for soine } n\right\}
$$

is the unique Cartan subalgebra of $\widetilde{J}$ containing $x$. If $k$ is algebraically closed then $\mathbb{E}=\Sigma \mathfrak{J}_{i i}$ with respect to a supplementary set of orthogonal primitive idempotents (cf. [3, pp. 343ff]).

Theorem 1. If $k$ is infinite then every Cartan subalgebra $(5)$ of $\mathfrak{J}$ contains regular elements and hence is of the form $\bigcap_{x}$ with $x$ regular.

Proof. First let $k$ be algebraically closed and $\mathcal{C}=\Sigma \mathfrak{S}_{i i}$ as above. By Lemma $3, H^{0}$. (S contains a dense open subset of $\mathfrak{S}_{\text {. Since }} \mathfrak{J}_{\mathrm{reg}}$ is invariant under all automorphisms of $\mathfrak{\Im}$ we have $\varnothing \neq\left(H^{0}\right.$. () $\cap \mathfrak{S}_{\text {reg }}=H^{0} \cdot\left(\sqrt{S} \cap \mathfrak{J}_{\text {reg }}\right)$, and $\sqrt{5}$ contains regular elements. If $k$ is not algebraically closed then ${ }^{S}{ }_{K}$ is a Cartan subalgebra of $\mathfrak{J}_{K}$ and therefore contains regular elements. Thus the function $c_{n-m}$ does not vanish on $\mathfrak{S}_{K}$, and since $k$ is infinite it does not vanish on $\sqrt{5}$.

Theorem 2. If $k$ is algebraically closed then any two Cartan subalgebras of $\mathfrak{J}$ are conjugate by an element of $H^{0}$.

Proof. Let $(\mathcal{S}$ and $(\mathcal{S}$ ' be Cartan subalgebras of $\Im$. By Lemma 3,

$$
\varnothing \neq\left(H^{0} \cdot(\mathfrak{S}) \cap\left(H^{0} \cdot \mathbb{C}^{\prime}\right) \cap \mathfrak{S}_{\mathrm{reg}}=H^{0} \cdot\left(\mathbb{S} \cap\left(H^{0} \cdot \mathbb{S}^{\prime}\right) \cap \mathfrak{S}_{\mathrm{reg}}\right)\right.
$$

Thus there exists a regular element $x \in \mathbb{S}^{\prime}$ and $h \in H^{0}$ such that $h(x) \in \mathbb{S}$. It follows that $h\left(\mathcal{S}^{\prime}\right)=h\left(\bigcap_{x}\right)=\bigcap_{h(x)}=\mathcal{S}$.

Theorem 3. Let $k$ be arbitrary. Then $\mathfrak{S}$ contains a Cartan subalgebra.

Proof. If $k$ is infinite then $\mathfrak{S}$ contains regular elements and therefore Cartan subalgebras. Let $k$ be finite and let $q$ be the number of elements of $k$. Then the Galois group of $K=k_{s}$ over $k$ is generated (topologically) by the Frobenius map $F: \lambda \mapsto \lambda^{q}$. If $X$ is an algebraic variety defined over $k$ we denote by $x \mapsto x^{(q)}$ the action of $F$ on $X$. Since $H^{0}$ is defined over $k$ (Lemma 2) the map $g \mapsto g^{-1} g^{(q)}$ from $H^{0}$ into itself is surjective [1, p. 369]. The action of $F$ on $\mathfrak{S}_{K}$ obviously staisfies

$$
(x+y)^{(q)}=x^{(q)}+y^{(q)}, \quad(\lambda x)^{(q)}=\lambda^{q} x^{(q)}, \quad \text { and } \quad(x y)^{(q)}=x^{(q)} y^{(q)} .
$$

Hence if $\left(\mathcal{S}\right.$ is a Cartan subalgebra of $\widetilde{J}_{K}$ so is $(\mathfrak{S}(q)$. By Theorem 2 there 
exists $g \in H^{0}$ such that $g(\mathbb{S}(q))=\mathbb{E}$, and $g=h^{-1} h^{(q)}$ for some $h \in H^{0}$. This implies $h(\mathbb{E})=h^{(q)}(\mathbb{E}(q))=h(\mathbb{S})^{(q)}$ and therefore $h(\mathbb{E})$ is defined over

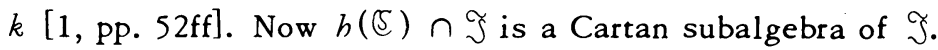

\section{BIBLIOGRAPHY}

1. A. Borel, Linear algebraic groups, Benjamin, New York, 1969. MR 40 \#4273.

2. D. M. Foster, Generalizations of nilpotence and solvability in universal classes of algebras, J. Algebra 26 (1973), 536-555.

3. N. Jacobson, Structure and representations of Jordan algebras, Amer. Math. Soc. Colloq. Publ., vol. 39, Amer. Math. Soc., Providence, R. I., 1968. MR 40 \#4330.

4. O. Loos, Jordan pairs, Springer Lecture Notes, Springer-Verlag, New York (to appear).

DEPARTMENT OF MATHEMATICS, UNIVERSITY OF BRITISH COLUMBIA, VANCOUVER, BRITISH COLUMBIA, CANADA VGT IWS 